\title{
COINTEGRATION AND CAUSALITY ANALYSIS BETWEEN FOREIGN DIRECT INVESTMENT, EXPORTS AND ECONOMIC GROWTH IN THE REPUBLIC OF CROATIA
}

\author{
Martina Sopta* \\ Vlatka Bilas ${ }^{* *}$ \\ Sanja Franc ${ }^{* * *}$
}

Received: 1. 10. 2021

Accepted: 29. 11. 2021

DOI https://doi.org/10.30924/mjcmi.26.2.8

\author{
Preliminary communication \\ UDC 339.727.22(497.5)“2000/2020“
}

\begin{abstract}
The main objective of this paper is to analyze the causal relationship between foreign direct investment (FDI), exports, and economic growth in the Republic of Croatia for the period 20002020 and determine the implications of research results on corporate management. The management of the investment enterprise is usually interested in high returns, whereas the management of the recipient enterprise is interested in higher productivity, spillovers, and larger market share on domestic and international markets. Several methodological approaches, including unit root tests, cointegration tests, and Granger causa-

tests indicated there is no long-term relationship between the real GDP growth rate, the share of $F D I$, and the share of exports of goods and services in real GDP. Based on the Granger causality test, it cannot be concluded that there is no causal relationship between the analysed variables. Finally, the paper discusses the implications of the conducted research for corporate management. The results indicate that managers are not discouraged by the fact that FDI is not correlated to economic growth, as investment decisions are determined by numerous factors and not primarily by the growth rate of a recipient country.
\end{abstract} lity test, were used to assess the relationship between gross domestic product (GDP) growth rate, on the one side, and the share of FDI and total exports of goods and services in real GDP, on the other side. The results of cointegration

\section{INTRODUCTION}

There are several sources of economic growth. Recent literature increasingly emphasizes the importance of innovation and technology, while an increase in production factors, exports of goods and services,
Keywords: foreign direct investment, export, gross domestic product, Croatia, corporate management

and FDI are taken as traditional sources of growth. The effects of FDI on the recipient economy have been discussed for decades and there is a common opinion that the effects of FDI inflows are predominantly positive. However, the empirical evidence

\footnotetext{
* Martina Sopta, PhD, associate professor, Faculty of Economics and Business, University of Zagreb, Trg J.F. Kennedy 6, 10000 Zagreb, Croatia, E-mail: msopta@efzg.hr, ORCID: https://orcid.org/0000-0001-7205-4989

** Vlatka Bilas, PhD, professor, Faculty of Economics and Business, University of Zagreb, Trg J.F. Kennedy 6, 10000 Zagreb, Croatia, E-mail: vbilas@efzg.hr, ORCID: https://orcid.org/0000-0002-9021-6651

${ }^{* * *}$ Sanja Franc, PhD, associate professor, Faculty of Economics and Business, University of Zagreb, Trg J.F. Kennedy 6, 10000 Zagreb, Croatia, E-mail: sfranc@efzg.hr, ORCID: https://orcid.org/0000-0001-8787-4429
} 


\section{Journal of Contemporary Management Issues}

is neither consistent nor unambiguous (see e.g. Nguyen, 2020; Abbes et al., 2015; Mahmoodi and Mahmoodi, 2016). The existence and the direction of a causal link between FDI and economic growth have not been unambiguously proven and it has been shown that the results depend on the sample, time period, and applied methodology.

The main objective of this paper is to research whether there is a causal link between FDI, exports, and economic growth on the example of the Republic of Croatia with the purpose of recognizing how this nexus between the selected variables affects investors and their investment decisions. Furthermore, the paper discusses the results of the conducted empirical research and implications for corporate management. Different methodological approaches were used to obtain robust results. The scientific contribution of the paper is reflected in a diversified methodological approach to the analysis of the correlation among FDI, exports, and economic growth, based on which conclusions can be made regarding the effects and importance of FDI.

The paper is divided into seven parts. After the introductory part follows the literature review. Then, a brief overview of the Croatian economy is given, after which the methodological approach is explained. In the fifth section, empirical results are presented and discussed. After that, implications for corporate management are addressed, and the final part is the conclusion.

\section{LITERATURE REVIEW}

As noted, research conclusions regarding the relationship between FDI, exports, and GDP vary greatly depending on the sample and the research methodology applied. In addition to international studies, a special emphasis in the literature review is given to the papers examining the case of Croatia.

Nguyen (2020) analyzed whether there is a correlation between FDI, financial aid, exports of goods and services, and economic growth. The empirical results showed that there is a positive relationship between net inflows of FD, financial aid, exports, and current GDP at a significance level of 1\%. Yilgor and Kokmaz (2019) analyzed how FDI impacted economic growth in the OECD countries. They used TodaYamamoto causality tests based on annual statistics for the period 1975-2008. The results confirm a causal relationship between GDP and FDI in France, Spain, and South Korea. In Greece, however, the direction of the causal relationship is reversed, from FDI to GDP. Ali and Hussain (2017) used the ARDL model to examine the impact of FDI on growth. Their research concluded that FDI has a positive impact on economic growth both in the short and the long run.

Furthermore, Abbes et al. (2015) analyzed the causal relationship between FDI and economic growth on the sample of 65 economies using cointegration and panel Granger causality tests. There was a disparity in the results as they indicated a oneway causality from FDI to GDP, which can be very informative for the decision on distribution of resources by sector to promote FDI. Basu et al. (2003) confirmed the existence of a bidirectional causality between economic growth and FDI in 23 observed developing countries for the period 19781996. The authors found that openness is an important factor that influences the direction of a causality relationship. More open countries have recorded causality in both directions, while in less open countries long-term causality predominantly goes from GDP to FDI. 
According to Bhatt (2013), who explored different dimensions of FDI and trade on the example of Vietnam, the cointegration test results indicated a long-run balanced relationship between exports, FDI, and GDP. Furthermore, FDI was found to be a significant variable, and the result showed that an increase in FDI of $1 \%$ will lead to an increase in exports of $0.25 \%$ with a time interval of one year. The Granger causality test showed that there is a unilateral relationship between exports and FDI and that the relationship moves from FDI to exports, meaning that FDI generates exports. Herzer (2008) re-examined the FDI growth hypothesis in 28 selected developing countries. Interestingly, the results indicated that in most countries, the relationship among the selected variables is not proven.

Regarding the literature review examining the case of Croatia, Jovančević (2007) analyzed the effects of FDI inflows in Croatia and other new members of the European Union and concluded that, in the long run, economic performance correlated with the amount of the FDI inflows, and national investment policies might af-fect the economic growth. Ivanović et al. (2014) concluded that FDI did not increase the level of Croatian competitiveness as expected because most of the FDI was brownfield and resulted in an employment decrease. They proposed measures to attract further investments, which would be more advantageous to the economy. Dritsaki and Stiakakis (2014) concluded in their research that FDI did not lead to growth in Croatia in the period 1994-2012, either in the short run or the long run. These results indicate that FDI does not have expected positive results for the Croatian economy. Toš Bublić et al. (2013) achieved similar results.

From selected CEE countries, Žiković et al. (2014) concluded there is a negative relationship between FDI and GDP growth only in Croatia. However, they found a long-run positive relationship between the imports-coverage ratio and GDP. On the other hand, Vukšić (2006) found that FDI positively impacted exports of 21 industry sectors in Croatia for the period 1996-2002, but the extent of the impact was low.

Bilas (2019) analyzed the effects of FDI on the GDP growth rate in Croatia using cointegration tests and the Granger causality test. She concluded that FDI does not have an effect on the GDP growth rate. The results coincide with this research, which is similar but broader in terms of the variables used and a more recent time period covered. Research in this paper leads to conclusions on the statistical relationship among the analyzed variables and based on that, a discussion on implications for corporate management is provided.

\section{CURRENT STATE OF THE CROATIAN ECONOMY}

Croatia is a post-transitional country that joined the EU in 2013. With the total GDP of 56.8 billion USD and at $65.2 \%$ of the EU27 GDP per capita in 2019, Croatia still lags behind its EU peers (World Bank, 2021). Croatian economy relies mostly on tourism; however, strategic development should rely on a more diversified industrial base, as the COVID-19 pandemic has proven. During the pandemic, all travel was suspended and the tourism sector was heavily hit. In 2020, Croatian GDP fell by $8.0 \%$, which was one of the largest falls among the EU members. The most significant cause of such a fall was the structure of the economy in which a large share is captured by tourism (Zubak and Hanzl, 2021). The consumption structure of GDP shows in more detail that the greatest impact on 


\section{Journal of Contemporary Management Issues}

the decline in economic activity was the decrease in foreign demand, i.e. the decline in the value of exports of goods and services.

According to the latest data of Croatian foreign trade statistics (CBS, 2021), in 2020, the level of exports was almost the same as in 2019 , i.e. exports fell by $0.7 \%$, while imports decreased by $6.9 \%$. Croatia's most important foreign trade partners are the EU member states, and in 2020 trade with the EU market accounted for about $70 \%$ of total exports and for about $80 \%$ of total imports. The total value of FDI from 1993 to 2020 amounted to 33.04 billion euros, an average of 1.18 billion euros per year or a total of 8,200 euros per capita. FDI amounted to 1.3 billion euros in 2020 , $5.5 \%$ more than the previous year and the most since 2010 (Zubak and Hanzl, 2021). Among all activities, the highest value of FDI in 2020 was realized in equity investments in real estate. After real-estate investments, the largest investments were recorded in financial services activities, the highest since 2010.

According to Mihaljek (2014), Croatia faces problems with the most important production resource - labour. Insufficient new jobs are created, and the educational structure of employees is improving too slowly. Technological progress is modest, which mirrors problems in higher education and scientific research systems, public administration, and research and development. The growth of the Croatian economy depends on investments, primarily in construction, but not in equipment and other service providers. In other words, economic growth is mainly based on construction, a distinctly cyclical activity whose dynamics largely depend on lending. Therefore, it is not surprising that GDP growth was strong in times of high foreign debt (2002-2008) and that the deep recession followed after the global financial crisis outbreak. Therefore, investments can be considered a significant growth factor, but there are deeper problems (Mihaljek, 2014) as the structure and sources of investment financing do not encourage a stable longterm economic growth.

\section{METHODS}

As already stated, this paper analyses whether FDI and exports have an impact on economic growth in Croatia and discusses whether this relationship affects investment flows. Empirical analysis includes several different methodological approaches to ensure statistical strength and robustness of the results. The robustness of the results was tested using the following three series: rtGDP, Log (FDI GDP), and Log (EXP GDP). The analysis based on these annual series was conducted for the period 2000-2020 and data were collected from the Croatian Bureau of Statistics (CBS) and Croatian National Bank (CNB). All three series are in current prices. The list and description of variables are provided by Table 1 . 
Table 1. Variables

\begin{tabular}{|l|l|}
\hline Series & Description \\
\hline rtGDP & LOG transformation of GDP due to negative growth rates (\%) (CBS) \\
\hline FDI_GDP & Foreign direct investments (CNB) (\% of GDP) (CBS) \\
\hline EXP_GDP & Exports of goods and services (CBS) (\% of GDP) (CBS). \\
\hline
\end{tabular}

Source: Authors

Empirical analysis has been conducted in three steps by performing: (1) unitroot tests, (2) cointegration tests, and (3) Granger causality test. Testing the stationarity, i.e. the changes in key statistical properties in variables over time and the existence of correlation among variables, is necessary for further modelling of series dynamics. Examining the correlation among the selected variables enables recognizing the implications of FDI, exports, and growth nexus for corporate management.

\section{RESULTS AND DISCUSSION}

Firstly, Dickey-Fuller (ADF) unit-root test and Phillips-Perron (PP) unit-root test, with and without a constant were applied. For Rothenberg and Stock Point Optimal (ERS) and Kwiatkowski-Phillips-SchmidtShin (KPSS) tests, only the offset test equation was evaluated (Tables 2 and 3 ).

Table 2. ADF, PP, KPSS and ERS unit-root tests on a series level

\begin{tabular}{|c|c|c|c|c|c|c|c|c|c|c|c|c|}
\hline \multirow[b]{2}{*}{ Series } & \multicolumn{4}{|c|}{ ADF } & \multicolumn{4}{|c|}{ PP } & \multicolumn{2}{|c|}{ KPSS } & \multicolumn{2}{|c|}{ ERS } \\
\hline & 訔 & 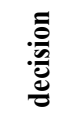 & U & 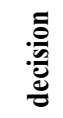 & 志 & 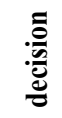 & U & 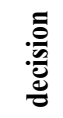 & U & 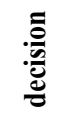 & U & 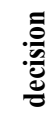 \\
\hline GDP & $\begin{array}{l}-2.24 \\
(.20)\end{array}$ & $\mathrm{I}(1)$ & $\begin{array}{l}-2.24 \\
(.20)\end{array}$ & I (1) & $\begin{array}{l}-2.24 \\
(.03)\end{array}$ & $\mathrm{I}(0)$ & $\begin{array}{r}-2.57 \\
(.11)\end{array}$ & I (1) & $\begin{array}{c}0.28 \\
(>.10)\end{array}$ & $\mathrm{I}(0)$ & $\begin{array}{c}3.95 \\
(>.10)\end{array}$ & $\mathrm{I}(1)$ \\
\hline FDI & $\begin{array}{l}-3.33 \\
(.03)\end{array}$ & $\mathrm{I}(0)$ & $\begin{array}{l}-3.33 \\
(.03)\end{array}$ & $\mathrm{I}(0)$ & $\begin{array}{l}-2.27 \\
(.03)\end{array}$ & $\mathrm{I}(0)$ & $\begin{array}{r}-3.37 \\
(.02)\end{array}$ & $\mathrm{I}(0)$ & $\begin{array}{c}0.25 \\
(>.10)\end{array}$ & I (1) & $\begin{array}{c}2.39 \\
(<.05)\end{array}$ & $\mathrm{I}(0)$ \\
\hline EXP & $\begin{array}{l}1.88 \\
(.34)\end{array}$ & $\mathrm{I}(1)$ & $\begin{array}{c}-1.88 \\
(.34)\end{array}$ & I (1) & $\begin{array}{r}-1.27 \\
(.94)\end{array}$ & I (1) & $\begin{array}{l}-1.88 \\
(.34)\end{array}$ & I (1) & $\begin{array}{c}0.67 \\
(<.05)\end{array}$ & $\mathrm{I}(0)$ & $\begin{array}{l}34.65 \\
(>.10)\end{array}$ & $\mathrm{I}(1)$ \\
\hline
\end{tabular}

Source: Authors 


\section{Journal of Contemporary Management Issues}

Table 3. ADF, PP, KPSS and ERS unit-roots tests - first differences

\begin{tabular}{|c|c|c|c|c|c|c|c|c|c|c|c|c|}
\hline \multirow[b]{2}{*}{ Series } & \multicolumn{4}{|c|}{ ADF } & \multicolumn{4}{|c|}{ PP } & \multicolumn{2}{|c|}{ KPSS } & \multicolumn{2}{|c|}{ ERS } \\
\hline & 咅 & 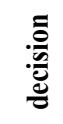 & U & 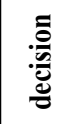 & 音 & 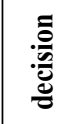 & U & 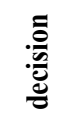 & U & 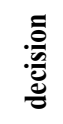 & U & 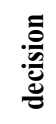 \\
\hline GDP & $\begin{array}{c}-4.51 \\
(<.01)\end{array}$ & $\mathrm{I}(0)$ & $\begin{array}{c}-4.46 \\
(<.01)\end{array}$ & I (0) & $\begin{array}{c}-4.45 \\
(<.01)\end{array}$ & I (0) & $\begin{array}{c}-4.46 \\
(<.01)\end{array}$ & I (0) & $\begin{array}{c}0.10 \\
(>.05)\end{array}$ & I (1) & $\begin{array}{c}3.44 \\
(>.05)\end{array}$ & $\mathrm{I}(1)$ \\
\hline FDI & $\begin{array}{c}-7.42 \\
(<.01)\end{array}$ & $\mathrm{I}(0)$ & $\begin{array}{l}-7.21 \\
(<.01)\end{array}$ & I ( $(0)$ & $\begin{array}{c}-7.72 \\
(<.01)\end{array}$ & I (0) & $\begin{array}{c}-7.49 \\
(<.01)\end{array}$ & I (0) & $\begin{array}{c}0.09 \\
(>.05)\end{array}$ & I (1) & $\begin{array}{c}3.33 \\
(>.05)\end{array}$ & $\mathrm{I}(1)$ \\
\hline EXP & $\begin{array}{c}-3.09 \\
(<.01)\end{array}$ & $\mathrm{I}(0)$ & $\begin{array}{r}-3.00 \\
(.05)\end{array}$ & I (0) & $\begin{array}{c}-3.09 \\
(<.01)\end{array}$ & I (0) & $\begin{array}{r}-3.05 \\
(.04)\end{array}$ & I $(0)$ & $\begin{array}{c}0.17 \\
(>.10)\end{array}$ & I (1) & $\begin{array}{c}6.10 \\
(>.10)\end{array}$ & $\mathrm{I}(1)$ \\
\hline
\end{tabular}

Source: Authors

Secondly, the newer generation of unit-root tests was applied, including PerronVogelsang, Clemente-Montañés-Reyes and Zivot-Andrews test (Tables 4 and 5).

Table 4. PV, ZA and CMR unit-roots tests

\begin{tabular}{|c|c|c|c|c|c|c|c|c|c|c|c|c|}
\hline \multirow[b]{2}{*}{ Series } & \multicolumn{3}{|c|}{$\begin{array}{c}\text { PV } \\
\text { (Additive Outlier) }\end{array}$} & \multicolumn{3}{|c|}{$\begin{array}{c}\text { ZA } \\
\text { (Additive Outlier) }\end{array}$} & \multicolumn{3}{|c|}{$\begin{array}{c}\text { CMR } \\
\text { (Additive Outlier) }\end{array}$} & \multicolumn{3}{|c|}{$\begin{array}{c}\text { CMR } \\
\text { (Innovative Outlier) }\end{array}$} \\
\hline & stat & BP & D & stat & BP & D & stat & BP & D & stat & BP & D \\
\hline & \multicolumn{12}{|c|}{ Series level } \\
\hline GDP & $\begin{array}{l}-3.03 \\
(.67)\end{array}$ & 2008 & $\mathrm{I}(1)$ & $\begin{array}{l}-3.44 \\
(>.10)\end{array}$ & 2009 & $\mathrm{I}(1)$ & -2.79 & 2010 & $\mathrm{I}(1)$ & -3.01 & 2006 & $\mathrm{I}(1)$ \\
\hline FDI & $\begin{array}{l}-6.15 \\
(<.01)\end{array}$ & 2014 & $\mathrm{I}(0)$ & $\begin{array}{l}-4.17 \\
(>.10)\end{array}$ & 2015 & $\mathrm{I}(1)$ & -4.38 & 2013 & $\mathrm{I}(0)$ & -11.5 & 2013 & $\mathrm{I}(0)$ \\
\hline \multirow[t]{2}{*}{ EXP } & $\begin{array}{l}-2.84 \\
(.77)\end{array}$ & 2009 & $\mathrm{I}(1)$ & $\begin{array}{l}-2.69 \\
(>.10)\end{array}$ & 2008 & $\mathrm{I}(1)$ & -2.86 & 2011 & $\mathrm{I}(1)$ & -23.2 & 2012 & $\mathrm{I}(0)$ \\
\hline & \multicolumn{12}{|c|}{ First differences } \\
\hline GDP & $\begin{array}{l}-5.37 \\
(<.01)\end{array}$ & 2009 & $\mathrm{I}(0)$ & $\begin{array}{l}-4.62 \\
(>.05)\end{array}$ & 2011 & $\mathrm{I}(1)$ & -3.78 & 2017 & $\mathrm{I}(0)$ & -57.6 & 2008 & $\mathrm{I}(0)$ \\
\hline FDI & $\begin{array}{l}-7.84 \\
(<.01)\end{array}$ & 2017 & $\mathrm{I}(0)$ & $\begin{array}{l}-8.77 \\
(<.01)\end{array}$ & 2017 & $\mathrm{I}(0)$ & -4.46 & 2013 & $\mathrm{I}(0)$ & -6.76 & 2014 & $\mathrm{I}(0)$ \\
\hline EXP & $\begin{array}{l}-4.10 \\
(.12)\end{array}$ & 2019 & $\mathrm{I}(1)$ & $\begin{array}{l}-3.64 \\
(>.10)\end{array}$ & 2010 & $\mathrm{I}(1)$ & -2.17 & 2017 & $\mathrm{I}(1)$ & -7.54 & 2008 & $\mathrm{I}(0)$ \\
\hline
\end{tabular}

Note. ${ }^{*} \mathrm{BP}$ - breaking point, $\mathrm{D}$ - decision.

Source: Authors 
Table 5. Clemente-Montañés-Reyes (CMR) unit-root test

\begin{tabular}{|l|c|c|c|c|c|c|c|c|}
\hline \multirow{2}{*}{ Series } & \multicolumn{7}{|c|}{ Innovative outlier } & \multicolumn{5}{c|}{ Additive outlier } \\
\cline { 2 - 9 } & $\boldsymbol{t}$-stat & $\mathbf{T B}_{\mathbf{1}}$ & $\mathbf{T B}_{\mathbf{2}}$ & decision & $\boldsymbol{t}$-stat & $\mathbf{T B}_{\mathbf{1}}$ & $\mathbf{T B}_{\mathbf{2}}$ & decision \\
\hline & \multicolumn{7}{|c|}{ Series level } \\
\hline GDP & -4.21 & 2007 & 2013 & $\mathrm{I}(1)$ & -3.42 & 2006 & 2014 & $\mathrm{I}(1)$ \\
\hline FDI & -4.62 & 2013 & 2017 & $\mathrm{I}(1)$ & 0.47 & 2012 & 2015 & $\mathrm{I}(1)$ \\
\hline EXP & -23.22 & 1998 & 2012 & $\mathrm{I}(0)$ & -4.11 & 2001 & 2014 & $\mathrm{I}(1)$ \\
\hline & \multicolumn{7}{|c|}{ First differences } \\
\hline GDP & -57.61 & 1998 & 2008 & $\mathrm{I}(0)$ & -4.29 & 2007 & 2013 & $\mathrm{I}(1)$ \\
\hline FDI & -31.53 & 2012 & 2014 & $\mathrm{I}(0)$ & -6.05 & 2008 & 2013 & $\mathrm{I}(0)$ \\
\hline EXP & -3.34 & 2006 & 2008 & $\mathrm{I}(1)$ & -2.88 & 2004 & 2009 & $\mathrm{I}(1)$ \\
\hline
\end{tabular}

Source: Authors

The CMR test with additive outliers, which takes into account sudden changes in series, revealed non-stationarity at the series level with two breaks. Therefore, it can be said that all series I(1) are with two structural breaks. If the time series are nonstationary, then the procedures for making it stationary need to be applied, which usually includes differentiating it or excluding the trend. The results of all unit-root tests performed are open for discussion because of the small size of the sample (20 observations). A small sample limits the strength of the unit-root test and the results should be taken carefully.

After stationarity of the series tests, the following step of the empirical research is performing cointegration texts: (1) EngleGranger cointegration test, (2) GregoryHansen cointegration test with a structural break, (3) Johansen cointegration test, (4) Bayer-Hanck meta cointegration test, and (5) the ARDL model. Results in Tables 6-8 indicate there is no long-term cointegration among the observed time series.

Table 6. Engle-Granger's cointegration test

\begin{tabular}{|l|c|c|c|c|}
\hline \multirow{2}{*}{$\begin{array}{l}\text { Dependent } \\
\text { variable }\end{array}$} & tau-stat & $\boldsymbol{P}$-value* & $\boldsymbol{z}$-stat & $\boldsymbol{P}^{*}$-value* \\
\cline { 2 - 5 } & \multicolumn{5}{|c|}{ series: Log (rGDP), Log (FDI/GDP) \& Log (EXP/GDP) } \\
\hline $\log (\mathrm{rGDP})$ & -1.66 & 0.86 & -7.17 & 0.72 \\
\hline $\log (\mathrm{FDI} / \mathrm{GDP})$ & -2.41 & 0.55 & -14.70 & 0.16 \\
\hline $\log (\mathrm{EXP} / \mathrm{GDP})$ & -0.83 & 0.98 & -2.04 & 0.98 \\
\hline \multicolumn{6}{|c|}{ With constant as an additional regressor } \\
\hline $\log (\mathrm{rGDP})$ & -1.66 & 0.90 & -7.17 & 0.79 \\
\hline $\log (\mathrm{FDI} / \mathrm{GDP})$ & -2.41 & 0.61 & -14.70 & 0.19 \\
\hline $\log (\mathrm{EXP} / \mathrm{GDP})$ & -0.83 & 0.99 & -2.04 & 0.99 \\
\hline
\end{tabular}

Source: Authors 


\section{Journal of Contemporary Management Issues}

Table 7. Gregory-Hansen cointegration test

\begin{tabular}{|c|c|c|c|c|c|c|c|c|c|c|c|}
\hline \multirow[t]{2}{*}{ Dependent } & \multirow{2}{*}{$\begin{array}{c}\text { Type } \\
\text { of } \\
\text { break }\end{array}$} & \multirow[t]{2}{*}{ ADF } & \multirow[t]{2}{*}{$Z_{t}$} & \multirow[t]{2}{*}{$Z_{a}$} & \multicolumn{3}{|c|}{ Break } & \multicolumn{3}{|c|}{$\begin{array}{l}5 \% \text { asymptomatic } \\
\text { critical values }\end{array}$} & \multirow[t]{2}{*}{$\begin{array}{l}\text { Cointe- } \\
\text {-gration } \\
\text { (Yes/No) }\end{array}$} \\
\hline & & & & & ADF & $Z_{t}$ & $Z_{a}$ & ADF & $Z_{t}$ & $Z_{a}$ & \\
\hline \multirow{3}{*}{$\begin{array}{l}\text { Log } \\
\text { (rGDP) }\end{array}$} & level & -4.31 & -4.42 & -26.84 & 2002 & 2002 & 2002 & -4.92 & -4.92 & -46.98 & $\begin{array}{c}\text { No (ADF, } \\
\left.Z_{t}, Z_{a}\right)\end{array}$ \\
\hline & trend & -5.46 & -5.60 & -25.97 & 1997 & 1997 & 1997 & -5.29 & -5.29 & -53.92 & $\begin{array}{l}\text { Yes (ADF, } \\
\left.Z_{t}\right) \text { No }\left(Z_{a}\right)\end{array}$ \\
\hline & regime & -4.70 & -4.70 & -25.85 & 2004 & 2002 & 2002 & -5.50 & -5.50 & -58.33 & $\begin{array}{c}\text { No (ADF, } \\
\left.Z_{t}, Z_{a}\right)\end{array}$ \\
\hline \multirow{3}{*}{$\begin{array}{l}\text { Log } \\
\text { (FDI/GDP) }\end{array}$} & level & -5.82 & -5.97 & -27.07 & 2012 & 2012 & 2012 & -4.92 & -4.92 & -46.98 & $\begin{array}{c}\text { Yes (ADF, } \\
\left.Z_{t}\right) \\
\text { No }\left(Z_{a}\right)\end{array}$ \\
\hline & trend & -6.21 & -6.37 & -28.22 & 2011 & 2011 & 2011 & -5.29 & -5.29 & -53.92 & $\begin{array}{c}\text { Yes (ADF, } \\
\left.Z_{t}\right) \\
\text { No }\left(Z_{a}\right)\end{array}$ \\
\hline & regime & -6.67 & -6.85 & -30.08 & 2008 & 2008 & 2008 & -5.50 & -5.50 & -58.33 & $\begin{array}{c}\text { Yes (ADF, } \\
\left.Z_{t}\right) \\
\text { No }\left(Z_{a}\right) \\
\end{array}$ \\
\hline \multirow{3}{*}{$\begin{array}{l}\text { Log } \\
\text { (EXP/ } \\
\text { GDP) }\end{array}$} & level & -3.55 & -3.82 & -16.96 & 2007 & 2011 & 2011 & -4.92 & -4.92 & -46.98 & $\begin{array}{c}\text { No (ADF, } \\
\left.Z_{t}, Z_{a}\right)\end{array}$ \\
\hline & trend & -5.73 & -5.87 & -27.66 & 1997 & 1997 & 1997 & -5.29 & -5.29 & -53.92 & $\begin{array}{c}\text { Yes (ADF, } \\
\left.Z_{t}\right) \\
\text { No }\left(Z_{a}\right) \\
\end{array}$ \\
\hline & regime & -4.04 & -4.06 & -16.74 & 2011 & 2011 & 2011 & -5.50 & -5.50 & -58.33 & $\begin{array}{c}\text { No (ADF, } \\
\left.Z_{t}, Z_{a}\right)\end{array}$ \\
\hline
\end{tabular}

Source: Authors.

Table 8. Johansen cointegration test

\begin{tabular}{|c|c|c|c|c|}
\hline $\mathbf{H}_{\mathbf{0}}$ & $\mathbf{H}_{\mathbf{1}}$ & Statistics test & $\mathbf{5 \%}$ critical value & $P$-value \\
\hline \multicolumn{5}{|c|}{ Trace statistics } \\
\hline & & 50.00 & 29.80 & $<0.01$ \\
\hline & 22.71 & 15.43 & $<0.01$ \\
\hline & 8.29 & 3.84 & $<0.01$ \\
\hline \multicolumn{5}{|c|}{ Maximum eigen value } \\
\hline & 20.29 & 21.13 & 0.07 \\
\hline & 14.41 & 14.26 & 0.05 \\
\hline & 8.29 & 3.84 & $<0.01$ \\
\hline
\end{tabular}

Source: Authors. 
Prior to evaluating the ARDL model, Fand t-tests were used to examine the existence of a long-run correlation of the three series. Using the Schwarz information criterion, the following ARDL model was specified: ARDL(2,0,2). The results are shown in Tables 9 and 10.

Table 9. F- and t- test results

\begin{tabular}{|c|c|c|c|c|c|c|c|c|}
\hline \multicolumn{5}{|c|}{$\boldsymbol{F}$ - test } & \multicolumn{4}{|c|}{$\boldsymbol{t}$ - test } \\
\hline Value & Significance & $\mathbf{I}(\mathbf{0})$ & $\mathbf{I}(\mathbf{1})$ & Significance & Value & $\mathbf{I}(\mathbf{0})$ & $\mathbf{I}(\mathbf{1})$ \\
\hline 0.19 & $10 \%$ & 3.44 & 4.47 & 0.70 & $10 \%$ & -2.57 & -3.21 \\
\hline & $5 \%$ & 4.27 & 5.47 & & $5 \%$ & -2.86 & -3.53 \\
\hline & $1 \%$ & 6.18 & 7.87 & & $1 \%$ & -3.43 & -4.10 \\
\hline
\end{tabular}

Source: Authors.

Table 10. Bayer-Hanck cointegration test

\begin{tabular}{|l|c|c|c|}
\hline \multirow{2}{*}{ Model } & \multicolumn{2}{|c|}{ Test } & With constant \\
\cline { 2 - 4 } & EG-JOH & EG-JOH-BO-BDM & Cointegration \\
\hline $\log (\mathrm{rGDP})=\mathrm{f}(\log (\mathrm{FDI} / \mathrm{GDP}), \log (\mathrm{EXP} / \mathrm{GDP}))$ & 3.64 & 4.07 & No \\
\hline $\log (\mathrm{FDI} / \mathrm{GDP})=\mathrm{f}(\log (\mathrm{rGDP}), \log (\mathrm{EXP} / \mathrm{GDP}))$ & 4.69 & 9.43 & No \\
\hline $\log (\mathrm{EXP} / \mathrm{GDP})=\mathrm{f}(\log (\mathrm{rGDP}), \log (\mathrm{FDI} / \mathrm{GDP}))$ & 3.44 & 5.38 & No \\
\hline & \multicolumn{3}{|c|}{ With constant and trend } \\
\hline $\log (\mathrm{rGDP})=\mathrm{f}(\log (\mathrm{FDI} / \mathrm{GDP}), \log (\mathrm{EXP} / \mathrm{GDP}))$ & 5.22 & 6.14 & No \\
\hline $\log (\mathrm{FDI} / \mathrm{GDP})=\mathrm{f}(\log (\mathrm{rGDP}), \log (\mathrm{EXP} / \mathrm{GDP}))$ & 2.56 & 4.17 & No \\
\hline $\log (\mathrm{EXP} / \mathrm{GDP})=\mathrm{f}(\log (\mathrm{rGDP}), \log (\mathrm{FDI} / \mathrm{GDP}))$ & 4.33 & 6.59 & No \\
\hline
\end{tabular}

Source: Authors.

Since there is no correlation between the (1995) Granger causality test was used series (Table 10), the Toda and Yamamoto (Table 11).

Table 11. Granger causality test

\begin{tabular}{|l|c|c|c|c|c|}
\hline \multirow{2}{*}{ Dependent variable } & \multicolumn{3}{|c|}{ Excluded series } & \multirow{2}{*}{ All series } & \multirow{2}{*}{ Causality } \\
\cline { 2 - 4 } & $\log ($ rGDP) & $\log ($ FDI/GDP) & $\operatorname{Log(EXP/GDP)~}$ & & \\
\hline $\log (\mathrm{rGDP})$ & - & $5.21(0.07)$ & $3.93(0.14)$ & $11.28(0.02)$ & No $\mid$ No $\mid$ Yes \\
\hline $\log (\mathrm{FDI} / \mathrm{GDP})$ & $6.22(0.04)$ & - & $8.16(0.02)$ & $8.39(0.08)$ & Yes $\mid$ Yes $\mid$ No \\
\hline $\log (\mathrm{EXP} / \mathrm{GDP})$ & $0.98(0.61)$ & $6.27(0.04)$ & - & $8.01(0.09)$ & No $\mid$ Yes $\mid$ No \\
\hline
\end{tabular}

Note. Chi-square statistics with P-values in parentheses are presented. The VAR (2) model was used.

Source: Authors

The examination of causality indicates there is no cause-and-effect relationship among the selected variables. As far as the share of exports of goods and services in real GDP and share of FDI are concerned, there is a bi-directional causal relationship both to the share of FDI and from the share 


\section{Journal of Contemporary Management Issues}

of FDI to the share of exports of goods and services in real GDP.

According to the empirical results, a causal link between the real GDP and FDI on the example of Croatia cannot be determined.

\section{IMPLICATIONS FOR CORPORATE MANAGEMENT}

There is a consensus in the literature that FDI has a positive impact on the recipient economy, but the scope of the positive effect depends upon different factors in the economy such as economic environment, social and political factors, as well as the institutional aspect (Oyegoke and Arasm, 2021).

Oyegoke and Arasm (2021) pointed out that FDI served many emerging countries in terms of earning foreign reserves via investments, although the literature is biased on the relationship between FDI and economic growth. Interestingly, Doeringer and Terkla (2012) found that the management of FDI recipient enterprises adopts management practices that promote productivity and foster social capital formation at the workplace. However, it can be concluded that positive effects from the FDI inflows outreach the scope of the research in this paper.

From the strategic management point of view, empirical research conducted in this paper can be observed from two different aspects: from the aspect of a recipient enterprise and from the aspect of an investment enterprise. The management of the investment enterprise is interested in high returns, while the management of the recipient enterprise is interested in higher productivity and production (if production is in question), better design, spillover of technology, knowledge, best managerial practices, larger market share, etc.
The results of the empirical research in this paper indicate that foreign investments and presence on foreign markets do not exert effects on national economic growth This can be significant for policymakers when designing regulation and investment incentive packages and determining exports subsidies. However, this conclusion is also interesting for corporate managers when deciding on the strategy of internationalization. Managers seem not to be discouraged by the fact that FDI is not correlated to economic growth as investment decisions are determined by numerous factors and not primarily by the growth rate of a recipient country (Ho et al., 2019). The choice of internationalization strategy also depends on the amount of profit. If transport and customs costs are high, FDI is more profitable than exports. Thus, if there are high transport costs or trade barriers, FDI is a preferable choice for entering foreign markets. Strategic decisions on FDI also depend on the level of ethical behaviour of enterprises and corporate governance structure in a recipient country (Appiah-Kubi et al., 2020). Appiah-Kubi et al. (2020) concluded that corporate governance independence from political influences is of major importance for obtaining positive FDI effects. In their research, Rajnoha et al. (2018) found that foreign-owned enterprises performed better than domestically owned ones. In addition to better results measured by typical business performance indicators, foreign-owned enterprises invested more in intangible assets and research, development, and innovation. Furthermore, managerial experience on foreign markets contributes to the success of FDI projects (Wenn et al., 2019). The business environment and regulatory framework influence the decision-making process regarding undertaking an FDI project (Vučković et al., 2002). Since emerging countries as Croatia do not have high growth rates and high purchasing power, 
market and resource-based determinants of FDI decisions can be of great importance. Emerging countries have proven to be interesting locations for foreign direct investors as they provide access to new markets, natural resources, low labour costs, and opportunities to avoid trade barriers (Asongu, 2018).

Moreover, depending on the type of FDI, it can positively affect domestic firms, either through spillovers, employment, trade, or by positively affecting domestic firms in their restructuring (Apostolov, 2016).

Although Croatia is one of the most successful countries in attracting FDI among the new EU members, nonetheless, when analyzing the structure of FDI inflows on the firm-level data it leads to less favourable conclusions (Bule and Ćudina, 2019). Most FDI is related to equity investments in the financial sector. FDI equity investments in other sectors are relatively modest and include investments in telecommunications, trade, and real estate. Recently, there have been new FDIs in the hotel industry, e.g. Liburnia rivijera, Bluesun, Valamar hotels, Tourist hotels, etc. and those are expected to have a positive impact on the national economy as they are projected to create new workplaces, offer new services and new opportunities for branding Croatia. It can be concluded that various factors affect the decision-making process for both domestic and foreign managers. While managers of domestic firms focus on benefiting from knowledge, skills, or technology spillovers and creating new jobs from FDI projects, foreign managers are more interested in the business environment, market size, and available resources in the recipient economy, among other things.

\section{CONCLUSION}

Based on the empirical analysis using the annual time series in the period from 2000 to 2020 , it can be concluded that FDI and exports of goods and services do not have a unequivocally statistically significant impact on real GDP growth in the Republic of Croatia. Research into this relationship is extremely important, especially in post-transition countries such as Croatia, because it can point to sources of growth and, consequently, to appropriate policies and measures to stimulate growth. On a firm level, investment and management decisions certainly depend upon the overall economic situation and on government policies and regulations, but they also depend on other internal factors and motives. Since FDIs have been increasing in Croatia in the past years, it can be concluded that they have been motivated by factors other than the national growth rate, given that it has been volatile. The limitation of the conducted analysis is the small sample size, i.e. short time series. This limitation largely explains the differences in conclusions based on the tests applied when testing the same hypothesis. However, this study can give insight into the relevance of certain macroeconomic factors important for corporate management and investment decisions.

\section{REFERENCES}

1. Abbes, S. M., Belmokaddem, M., Guellil, M. S., Ghouali Y. Z. (2015). Causal Interactions between FDI, and Economic Growth: Evidence from Dynamic Panel Co-Integration. Procedia Economics and Finance, 23, $276-290$.

2. Ali, N., Hussain, H. (2017). Impact of Foreign Direct Investment on the Economic Growth of Pakistan. 


\section{Journal of Contemporary Management Issues}

American Journal of Economics, 7(4), 163-170.

3. Appiah-Kubi, S. N. K., Malec, K., Maitah, M., Kutin, S. B., Pankova, L., Phiri, J., Zaganjori, O. (2020). The Impact of Corporate Governance Structures on Foreign Direct Investment: A Case Study of West African Countries. Sustainability, 12, 3715, 1-15.

4. Apostolov, M. (2016). The Role of Foreign Direct Investments in Southeastern Europe. Revue d'études comparatives Est-Ouest, 2016/3 (47), $45-82$.

5. Asongu, S., Ahpak, U., Isihak, S. (2018). Determinants of foreign direct investment in fast-growing economies: evidence from the BRICS and MINT countries. Financial Innovation, 4(26).

6. Basu, P., Chakraborty, C., Reagle, D. (2003). Liberalisation, FDI, and growth in developing countries: a panel co-integration approach. Economic Inquiry, 51, 510-516.

7. Bilas, V. (2019). Foreign direct investment and economic growth relationship in Croatia. UTMS Journal of Economics, 10(2), 175-187.

8. Bhatt, P.R. (2013). Causal relationship between exports, FDI and income: the case of Vietnam. Applied Econometrics and International Development, 13-1.

9. Bule, M., Ćudina, A. (2019). Foreign Direct Equity Investments and Foreign Ownership Premium: The Case of Croatia. Working Papers W-58. Croatian National Bank.

10. Croatian Bureau of Statistics (2021). Database. Available at: https://www. dzs.hr/ (November 1, 2021).

11. Doeringer, P. B., Terkla, D. G. (2012). Foreign Direct Investment, Management Practices, and Social
Capital: New Evidence on the Host Country Effects of Japanese Multinationals. Available at: http:// www.bu.edu/econ/files/2012/11/dp129. pdf (October 17, 2021).

12. Dritsaki, C., Stiakakis, E. (2014). Foreign Direct Investments, Exports, and Economic Growth in Croatia: A Time Series Analysis. Procedia Economics and Finance, 14, 181-190.

13. Herzer, D. (2008). The long run relationship between outward FDI and domestic output: evidence from panel data. Economic Letters, 100(1), 146-149.

14. Ho, T., Nga, V.T., Doung, V. H. (2019). The determinants of foreign direct investment in ASEAN: New evidence from financial integration factor. Business and Economic Horizons, 15(2), 292-303.

15. Ivanovic, Z., Bogdan, S., Baresa, S. (2014). Impact of foreign direct investments on Croatian financial growth. UTMS Journal of Economics, 5(2), 141-150.

16. Jovančević, R. (2007). The impact of foreign direct investment flows on Croatian economy - a comparative analysis. Ekonomski pregled, 58(12), 826-850.

17. Mahmoodi, M., Mahmoodi, E. (2016). Foreign direct investment, exports, and economic growth: Evidence from two panels of developing countries. Economic Research, 29(1), 938-949.

18. Mihaljek, D. (2014). Izvori rasta u Hrvatskoj. Bank for International Settlements. Available at: https:// www.eizg.hr/UserDocsImages/O NAMA/klub-eiz-a/kako-pobuditi-rasthr-gospodarstva29914/Dubravko MihaljekIzvori_rasta_u_Hrvatskoj.pdf (November 1, 2021) 
19. Nguyen, C. H. (2020). The Impact of Foreign Direct Investment, Aid and Exports on Economic Growth in Vietnam. Journal of Asian Finance, Economics and Business, 7(10), 581589.

20. Oyegoke, E. O., Arasm, O. N. (2021). Impact of Foreign Direct Investment on Economic Growth in Nigeria. MPRA Paper No. 108348. Available at: https:// mpra.ub.uni-muenchen.de/108348/1/ MPRA_paper_108348.pdf (October 17, 2021).

21. Rajnoha, R., Merkova, M., Dobrovič, J., Rozsa, Z. (2018). Business performance management and FDI: key differences between foreign and domestic-owned firms - a case of Slovakia. Journal of Business Economics and Management, 19(1): 42-62.

22. Toda, H. Y., Yamamoto, T. (1995). Statistical inference in vector autoregressions with possibly integrated processes. Journal of Econometrics, 66, 225-250.

23. Toš Bublić, T., Pavičić, N., Rešetar, Z. (2013). FDI and economic growth in Croatia according to economic theory, Dubrovnik International Economic Meeting, 1(1). Available at: https:// hrcak.srce.hr/161427 (November 1,2021).

24. Vučković, M., Bobek, V., Maček, A., Skoko, H., Horvat, T. (2020). Business environment and foreign direct investments: the case of selected European emerging econo-mies. Ekonomska istraživanja, 33(1), 243-266.

25. Vukšić, G. (2006). Impact of foreign direct investment on Croatian manufacturing industry. Institute of Public Finance, Occasional Paper No. 27.

26. Wen, W., Ke, Y., Sun, X. (2019). Managerial Foreign Experience and Outward Foreign Direct Investment: Evidence from China. Asian Growth Research Institute.

27. World Bank (2021). Overview: Croatia. Available at: https://www.worldbank. org/en/country/croatia/overview\#1 (1.11.2021).

28. Yilgor, M., Korkmaz, S. (2019). Causality Relationship between Foreign Direct Investment and Economic Growth. The Empirical Economics Letters, 10(8), 786-792.

29. Zubak, D., Hanzl, Ž. (2021). Hrvatsko gospodarstvo 2020. godine. Zagreb: HGK.

30. Žiković, S., Tomas Žiković, I., Grdinić, M. (2014) Detangling growth, exports, imports and FDI knot in selected CEE countries. EFRI Exclusive Working Papers 2014-001. Available at: https://www.efri.uniri.hr/upload/ EFRI\%20EXCLUSIVE\%20papers/ Exclusive\%20EFRI\%20Zikovic\%20 Tomas\%20Grdinic_2014_01.pdf (November 1, 2021). 


\title{
Journal of Contemporary Management Issues
}

\author{
KOINTEGRACIJA I ANALIZA KAUZALNOSTI \\ IZRAVNIH STRANIH INVESTICIJA, IZVOZA I \\ EKONOMSKOG RASTA U REPUBLICI HRVATSKOJ
}

\begin{abstract}
Sažetak
Temeljni cilj ovog rada je analizirati uzročno-posljedičnu povezanost izravnih stranih investicija, izvoza i ekonomskog rasta u Republici Hrvatskoj, za razdoblje 2000-2020 te utvrditi implikacije dobivenih istraživačkih rezultata za menadžment u poslovnom sektoru. Menadžment investicijskih društava je obično zainteresiran za visoke povrate, dok su primatelji sredstava obično zainteresirani za postizanje više razine produktivnosti, efekte prelijevanja, kao i povećanje tržišnog udjela na domaćem i međunarodnom tržištu. Nekoliko metodoloških pristupa, uključivši i testove stacionarnosti, kointegracije te Grangerov test kauzalnosti, korišteno je za utvrđivanje odnosa između stope rasta bruto domaćeg proizvoda (BDP) s udjelima izravnih stranih investicija te ukupnog izvoza proizvoda i usluga u realnom BDP-u. Rezultati kointegracijskih testova ukazuju na nepostojanje dugoročnog odnosa između analiziranih varijabli. Na kraju rada se diskutiraju implikacije provedenog istraživanja za poslovni menadžment. Rezultati istraživanja ukazuju da se menadžeri ne moraju voditi činjenicom da izravne strane investicije nisu povezane s ekonomskim rastom, s obzirom da na investicijske odluke djeluju $i$ različiti čimbenici, koji nisu povezani sa stopom rasta zemlje-primateljice ulaganja.
\end{abstract}

Ključne riječi: izravne strane investicije, izvoz, bruto domaći proizvod, Hrvatska, poslovni menadžment 